\title{
Amplitude of low-frequency fluctuation (ALFF) may be associated with cognitive impairment in schizophrenia: a correlation study
}

Pengshuo Wang ${ }^{1,2}$, Jian Yang ${ }^{4}$, Zhiyang Yin ${ }^{1,2}$, Jia Duan ${ }^{1,2}$, Ran Zhang ${ }^{1,2}$, Jiaze Sun ${ }^{1,2}$, Yixiao Xu ${ }^{1,2}$, Luyu Liu $^{1,2}$, Xuemei Chen ${ }^{1,2}$, Huizi Li ${ }^{1,2}$, Jiahui Kang ${ }^{1,2}$, Yue Zhu ${ }^{1,2}$, Xin Deng ${ }^{1,2}$, Miao Chang ${ }^{4}$, Shengnan Wei ${ }^{2,4}$, Yifang Zhou ${ }^{1,2}$, Xiaowei Jiang ${ }^{2,4}$, Fei Wang ${ }^{1,2,4^{*}}$ and Yanqing Tang ${ }^{1,2,3^{*}}$ (D)

\begin{abstract}
Background: Cognitive impairments are prominent in schizophrenia (SZ). Imaging studies have demonstrated that functional changes of several areas of the brain exist in SZ patients. The relationships between these two indexes are largely unexplored in SZ. The MATRICS Consensus Cognitive Battery (MCCB) was used to measure cognitive impairment in multi-dimensional cognitive fields of SZ patients. This study was conducted to explore the relationship between cognitive functional impairment and the amplitude of low-frequency fluctuation (ALFF) in SZ patients.

Method: A total of 104 participants (44 SZ patients and 60 age- and gender-matched healthy controls (HC)) were recruited for this study. The MCCB was used to assess cognitive function of the participants, while brain activity was assessed using the ALFF. The relationship between the MCCB and the ALFF was investigated by using a correlation analysis.
\end{abstract}

Results: There were significant differences between SZ patients and HC in MCCB total and domain scores as well as in ALFF results. The reduction of ALFF in the bilateral postcentral gyri and paracentral lobule in SZ patients has a negative correlation with the MCCB sub-test of symbol coding.

Conclusion: These findings suggest that the reduction of ALFF in bilateral postcentral gyri and paracentral lobule may be related to cognitive impairment in SZ patients.

Keywords: Schizophrenia, MCCB, ALFF

\section{Background}

The etiology of schizophrenia (SZ) as a mental disorder remains unclear. SZ has a heterogeneous clinical manifestation, and cognitive impairment presents as a common, prominent symptom [1-3]. Several studies have reported multiple cognitive impairments in SZ patients,

\footnotetext{
* Correspondence: fei.wang@cmu.edu.cn; tangyanqing@cmu.edu.cn 'Department of Psychiatry, The First Affiliated Hospital of China Medical University, Shenyang, Liaoning 110001, People's Republic of China Full list of author information is available at the end of the article
}

including deficiencies in working memory, executive function, attention, processing speed, and social cognition $[4,5]$. Additional areas of cognitive function, such as theory of mind [6] and autobiographical memory [7], may also be affected. The assessment of cognitive impairment is extremely important, as it may impact the processes of diagnosis, therapy, and rehabilitation [8].

The US National Institute of Mental Health (NIMH) developed the Measurement and Treatment Research to Improve Cognition in Schizophrenia (MATRICS) initiative to stimulate the development of new drugs that address the cognitive deficits associated with schizophrenia

(C) The Author(s). 2019 Open Access This article is distributed under the terms of the Creative Commons Attribution 4.0 International License (http://creativecommons.org/licenses/by/4.0/), which permits unrestricted use, distribution, and 
[9]. To accomplish this goal, the MATRICS consensus cognitive battery (MCCB) was developed, and it is currently widely used $[10,11]$. The measurement of the cognitive impairment of SZ patients is estimated based on test-retest reliability, high utility as a repeated measure, relationship to functional outcome, potential changes in response to pharmacological agents, tolerability, and practicality $[9,12]$. Based on previous research and input from experts, seven cognitive domains, including working memory, speed of processing, social cognition, attention-vigilance, verbal learning, visual learning, reasoning and problem-solving, were divided into 10 subtests. Several studies reported significant impairment in each of the seven MCCB domains in SZ patients compared to healthy controls (HC) [13-15]. After the MCCB was translated into Chinese, the clinical reliability and validity of MCCB were established between SZ patients and HC [16] to allow for its widespread use in cognitive research in SZ patients in China [17-19].

The resting state functional magnetic resonance imaging ( $\mathrm{R}-\mathrm{fMRI}$ ) technique is a noninvasive and advanced neuroimaging measurement that has been recently used to investigate the pathophysiology of several disorders. The amplitude of low-frequency fluctuation (ALFF) [20] is an R-fMRI indicator that is used to detect the regional intensity of spontaneous fluctuations in the BOLD signal, which pinpoints the spontaneous neural activity of specific regions and physiological states of the brain. Electrophysiological studies [21] have shown that low-frequency oscillations may arise from spontaneous neuronal activity, which is of physiological significance and manifests in the rhythmic activity of the brain region through the interaction of information between connected brain regions; therefore, ALFF may reflect the characteristics of the brain [22]. ALFF studies suggested that intrinsic resting-state activity promotes or allows specific brain circuits to participate in cognitive tasks, and resting activity predicts subsequent task-induced brain responses and behavioral performance [23-25]. ALFF measures brain activity without cognitive load, and brain abnormalities in this state that correlate with cognition may be the basis of SZ cognitive impairment; therefore, we used ALFF in R-fMRI to examine SZ patients. Recent studies have reported gray matter ALFF changes in SZ patients. For example, Hoptman et al. [26] demonstrated that SZ patients, compared to $\mathrm{HC}$, exhibited lower ALFF in the cuneus/precuneus, precentral gyrus, and lingual gyrus and exhibited higher ALFF in the left hippocampus/parahippocampus. A meta-analysis reported that SZ patients showed decreased ALFF in the bilateral occipital, posterior parietal cortices, sensorimotor cortex, and right superior temporal gyrus and elevated ALFF in the bilateral striatum, medial temporal cortex, medial prefrontal cortex, and lateral orbitofrontal cortex compared to that of HC [27]. Alonso-Solís et al. [28] revealed that SZ patients with auditory verbal hallucinations showed increased ALFF values in the bilateral temporal pole and parahippocampal gyrus and decreased ALFF values in the occipital pole, lingual gyrus, precuneus, and cingulate cortex. Non-hallucinating SZ patients showed increased ALFF values in the temporal fusiform and parahippocampal gyrus and decreased ALFF in the occipital lobe and precuneus. Zheng et al. [29] found that adolescents with early-onset SZ showed significantly increased ALFF in the bilateral orbitofrontal cortex and significantly decreased ALFF in the ventral precuneus. Another study reported that a first-episode SZ group showed significantly decreased ALFF in the orbital/medial frontal lobe and significant increases in ALFF in the left and right putamen [30].

Several researchers reported that SZ was accompanied with an impairment in cognitive domains, and the ALFF values in multiple brain regions were altered compared to those of HC. To the best of our knowledge, no previous study has investigated the relationship between cognitive impairment and changes in ALFF values in SZ patients. Therefore, the present study examined whether cognitive impairments in SZ patients were associated with ALFF values, as assessed by the MCCB and by R-fMRI. We hypothesized that cognitive function tested with the MCCB would be associated with ALFF in the brain regions that show changes during schizophrenia; for instance, working memory and social cognition may be associated with ALFF in the prefrontal cortex and information processing speed may be associated with ALFF in the postcentral gyri and paracentral lobule.

\section{Materials and methods Participants}

Forty four patients who met DSM-IV criteria (American Psychiatric Association, 2000.) were recruited from the Department of Psychiatry, Frist Affiliated Hospital of China Medical University and the inpatient department of Mental Health Center of Shenyang, during the study, 41 patients were treated with medication, while the remaining 3 were not. $60 \mathrm{HC}$ were recruited by advertisement from the community. Inclusion criteria for the study are as follows: 1) must be between the ages of 16-45, must be diagnosed with SZ according to DSM-IV-TR standards (at least two of the following symptoms: hallucinations, delusions, disorganized speech and catatonic or bizarre behavior and negative symptoms; symptoms persist for at least 1 month and continuous signs more than 6 months; social function impairment; exclude schizoaffective /mood disorder 
and substance abuse), and the diagnoses must be confirmed by 2 trained psychiatrists using the Structured Clinical Interview for DSM-IV Axis I Disorders (SCID-I); 2) HC subjects must not have current or life time Axis I Disorders, nor could they have any first-degree relatives with a history of Axis I disorders. Exclusion criteria for the study are as follows: 1) must not have a history of major physical disorders, particularly those that may be associated with brain tissue changes such as hypertension, diabetes, or metastatic disease; 2) must not have: unstable diseases such as heavy asthma; neurological abnormalities, including major head trauma (loss of consciousness lasting more than 5 min), epilepsy, cerebrovascular disease, brain tumors, or neurodegenerative diseases; somatic diseases that may cause mood disorders such as multiple sclerosis, thyroid disease, etc.; 3) no MRI contraindication; and 4) no lifetime or current substance dependence or abuse. All participants signed informed consent as approved by the Ethics Committee of China Medical University.

\section{Cognitive assessment}

Assessment of neurocognitive functioning was completed using the MATRICS Consensus Cognitive Battery (MCCB). This instrument includes 10 tasks across 7 cognitive domains, including: speed of processing (Brief Assessment of Cognition in Schizophrenia Symbol Coding, Category Fluency, Trails A), attention-vigilance (Continuous Performance Test), working memory (WMS-III Spatial Span, Letter Number Span), verbal learning (Hopkins Verbal Learning Test - Revised), visual learning (Brief Visuospatial Memory Test - Revised), reasoning and problem solving (The Mazes test), and social cognition (Mayer - Salovey - Caruso Emotional Intelligence Test). The MCCB yields 10 subscale scores, 7 domain scores, and a composite score, calculated across the 7 domains. The MCCB shows good test-retest reliability (with scores ranging from $r=0.69$ to $r=0.85$ ), practicality, and tolerability. The collective battery took approximately $60-90 \mathrm{~min}$ to administer. In this study, all patients with SZ and $\mathrm{HC}$ completed the MCCB.

\section{Image acquisition}

Scanning took place on the $3 \mathrm{~T}$ MRI scanner (General Electric, Milwaukee, USA) at the Image Institute of First Affiliated Hospital of China Medical University, Shenyang, China. Earplugs and foam pads were used to minimize scanner noise and head motion. A standard head coil was used for radio frequency transmission and reception of the nuclear magnetic resonance signal. Functional images were collected using a gradient echo planar imaging (EPI) sequence (a 6 min 40 s resting state
fMRI scan, TR $=2000 \mathrm{~ms}, \mathrm{TE}=40 \mathrm{~ms}, \mathrm{FOV}=24 \mathrm{~cm} \times 24$ $\mathrm{cm}$, flip angle $=90^{\circ}$, matrix $=64 \times 64$, slices $=35$, slice thickness $=3 \mathrm{~mm}$, no gap). Participants were instructed to close their eyes, remain awake, and keep their mind blank during the resting state scan (after the scanning we checked this with the subjects).

\section{Image data processing}

Image data processing was performed by using Data Processing Assistant for Resting-State fMRI (DPABI, 2.3, Advanced edition) [31]. We discarded the initial 10 scan volumes to allow for steady-state magnetization. Then corrected for slice timing and head motion (using a least squares approach and a six-parameter spatial transformation). We excluded participants whose head motion exceeded $3 \mathrm{~mm}$ or rotation that exceeded $2.5^{\circ}$ during scanning. The standard Montreal Neurological Institute (MNI) template provided by SPM was then used for spatial normalization with a resampling voxel size of $3 \mathrm{~mm} \times 3 \mathrm{~mm} \times 3 \mathrm{~mm}$. The images were spatially smoothed with a 6-mm full-width at half-maximum Gaussian filter. We conducted time series linear detrending and temporal band-pass filtering $(0.01-0.08 \mathrm{~Hz})$ to remove low-frequency drifts and physiological high-frequency noise.

The ALFF analysis was carried out using DPABI. The filtered time series of each voxel was transformed into the frequency domain with a Fast Fourier Transform and the power spectrum was then obtained. We were measured ALFF by obtaining the square root of the signal across $0.01-0.08 \mathrm{~Hz}$ for each voxel [20]. For standardization purposes and to reduce the influence of individual variation in ALFF values, the ALFF of each voxel was further divided by the global mean of ALFF values for each subject within the default brain mask from the DPABI, with background and other non-brain tissue signals removed. This created a standardized whole-brain ALFF map.

\section{Statistical analysis}

We performed two-sample t-tests on MCCB scores between the two groups, and compared the differences in total score and each sub-test score, the significant level was set at $p<0.05$. Two-sample t-tests were used to compare ALFF data between the SZ patients and HC using DPABI to found significant brain regions, the significant level was set at $p<0.001$ (GRF, Gaussian random field corrected).

\section{Analysis of associations between ALFF and MCCB scores}

To explore the detailed associations between ALFF and MCCB scores in time, the following analysis was conducted, ALFF values were extracted from the 
Table 1 Demographic data of all subjects

\begin{tabular}{|c|c|c|c|c|}
\hline & $S Z(n=44)$ & $\mathrm{HC}(n=60)$ & $t / x^{2}$ & $p$ \\
\hline Age (years, mean \pm S.D.) & $25.00 \pm 7.49$ & $30.13 \pm 8.49$ & -3.260 & 0.250 \\
\hline Sex (female/male) & $31 / 13$ & $37 / 23$ & 0.352 & 0.866 \\
\hline Education (years, mean \pm S.D.) & $14.35 \pm 3.73$ & $12.52 \pm 3.05$ & 2.659 & 0.009 \\
\hline Medication (Y/N) & $41 / 3$ & N/A. & & \\
\hline First episode $(\mathrm{Y} / \mathrm{N})$ & $23 / 21$ & N/A. & & \\
\hline Duration (month, mean \pm S.D. $)^{a}$ & $43.02 \pm 42.42$ & N/A. & & \\
\hline Medication-CPZ Equivalent & $365.373 \pm 150.8$ & N/A. & & \\
\hline BPRS (total, mean \pm S.D. $)^{\text {a }}$ & $27.25 \pm 9.13$ & $18.46 \pm 1.57$ & 7.315 & $<0.001$ \\
\hline
\end{tabular}

Note: SD Standard deviation, $Y$ Yes, $N$ No, N/A None, BPRS Brief Psychiatric Rating Scale

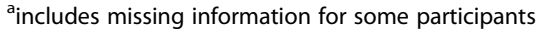

In the SZ group, 40 were taking atypical antipsychotics including clozapine $(y=0.6903 x+69.747)$, risperidone $(y=0.0116 x+0.0446)$, olanzapine $(y=0.0332 x+$ 2.0093), aripiprazole $(y=0.0266 x+5.311)$, ziprasidone $(y=0.1649 x+46.134)$ and quetiapine $(y=0.9004 x+85.459)$ chlorpromazine is represented by " $x$ " in the above formulas, 1 was taking paroxetine, and 3 were not taking any psychotropic medication at the time of scan. All antipsychotic doses were converted to chlorpromazine equivalents using standard procedures, added to demographic data

significant regions. The correlation coefficient between the significant ALFF values in significant regions and MCCB scores in SZ group was calculate using SPSS (Statistical Product and Service Solutions) 22.0 software (SPSS Inc., Chicago, Illinois) software, and results were corrected by False Discovery Rate (FDR) correction $(\mathrm{q}<0.05)$.

\section{Results}

\section{Demographic data outcome}

There were no significant differences between SZ and HC groups in age $(p=0.250)$ or gender $(p=0.866)$, antipsychotic drug doses were converted to chlorpromazine equivalent [32]. The results of demographic data were listed in Table 1.

\section{ALFF group differences}

ALFF analysis yielded 10 clusters, that includes 12 regions. We used Anatomical Automatic Labeling template to identify the brain regions in the clusters using DPABI software. The SZ group showed significantly decreased ALFF in the bilateral lingual, calcarine, cuneus, postcentral, paracentral lobule, and right precuneus. Furthermore, the SZ group had significantly increased ALFF in the bilateral caudate, orbital part of the inferior frontal gyrus, middle frontal gyrus, medial superior frontal gyrus, triangular inferior frontal gyrus, and superior frontal gyrus (Fig. 1, Table 2), in the figure, the yellow area shows that the ALFF value of SZ is higher than HC, and the blue area shows that the ALFF value of SZ is lower than $\mathrm{HC}$.

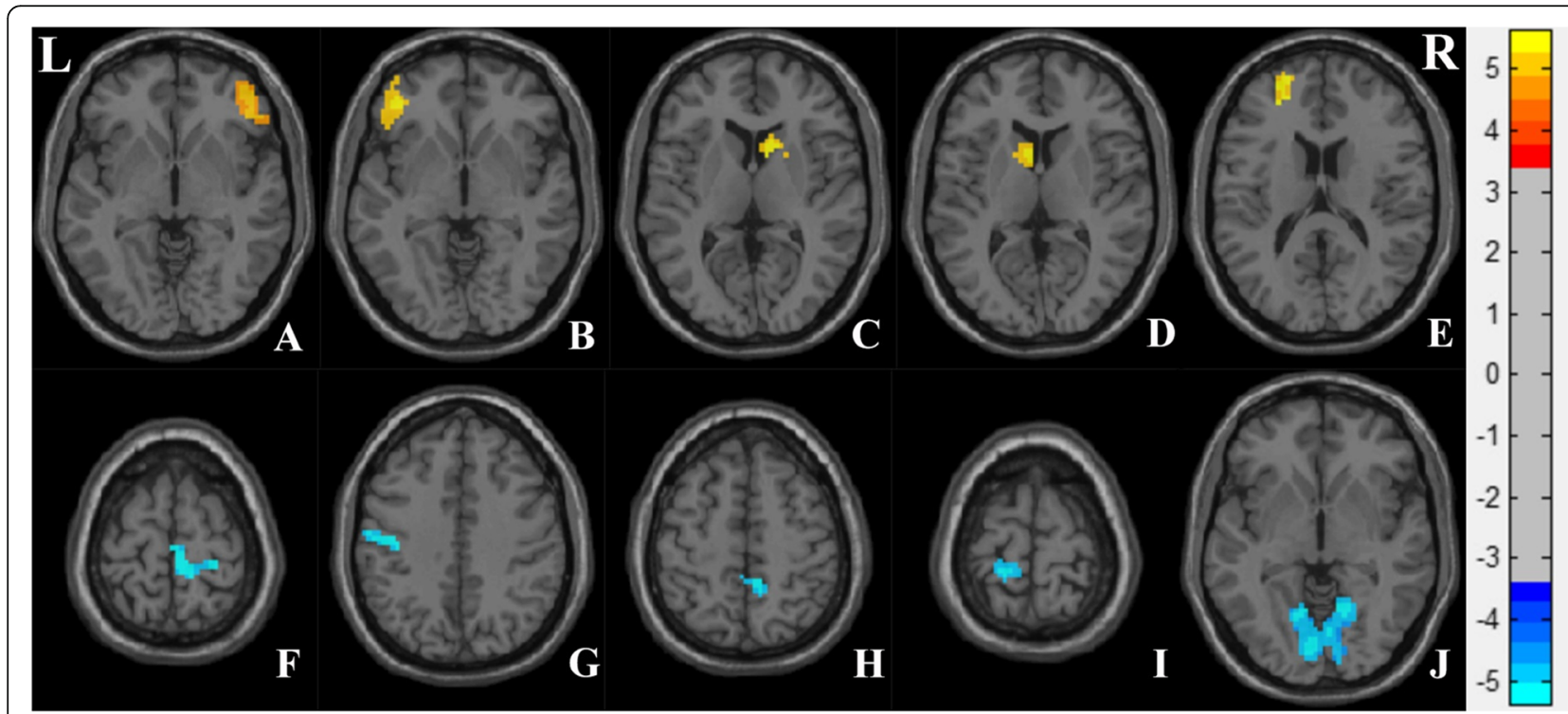

Fig. 1 Regions showing altered amplitude of low frequency fluctuation (ALFF) in the SZ group, compared to the HC group. The color bar represents the range of $t$ values. $L$, left; $R$, right 
Table 2 Regions with ALFF differences in SZ and HC subjects

\begin{tabular}{|c|c|c|c|c|c|c|}
\hline & \multirow[t]{2}{*}{ Brain regions } & \multirow{2}{*}{$\begin{array}{l}\text { Cluster } \\
\text { size }\end{array}$} & \multicolumn{3}{|c|}{ Peak coordinates (MNI) } & \multirow{2}{*}{$\begin{array}{l}\text { T } \\
\text { values }\end{array}$} \\
\hline & & & $x$ & y & z & \\
\hline \multirow[t]{12}{*}{$\mathrm{SZ}>\mathrm{HC}$} & A L Medial superior frontal gyrus & 237 & 51 & 27 & 6 & 5.6597 \\
\hline & R Middle frontal gyrus & 151 & & & & \\
\hline & R Medial superior frontal gyrus & 127 & & & & \\
\hline & R Superior frontal gyrus & 123 & & & & \\
\hline & R Pars triangularis inferior frontal gyrus & 94 & & & & \\
\hline & R Orbital inferior frontal gyrus & 82 & & & & \\
\hline & L Superior frontal gyrus & 62 & & & & \\
\hline & B L Pars triangularis inferior frontal gyrus & 82 & -39 & 42 & -9 & 5.0682 \\
\hline & L Orbital inferior frontal gyrus & 58 & & & & \\
\hline & C R Caudate & 75 & 12 & 12 & 6 & 5.0632 \\
\hline & D L Caudate & 79 & -9 & 9 & 3 & 5.0506 \\
\hline & E L Medial frontal gyrus & 40 & -24 & 54 & 18 & 4.4804 \\
\hline \multirow[t]{12}{*}{$\mathrm{SZ}<\mathrm{HC}$} & F R Paracentral lobule & 36 & 12 & -36 & 69 & -4.2799 \\
\hline & R Postcentral gyrus & 24 & & & & \\
\hline & GL Postcentral gyrus & 136 & -51 & -12 & 33 & -4.3341 \\
\hline & H R Precuneus & 41 & 9 & -48 & 51 & -4.5002 \\
\hline & I L Postcentral gyrus & 38 & -18 & -39 & 72 & -4.9716 \\
\hline & L Paracentral lobule & 23 & & & & \\
\hline & J L Calcarine & 200 & 6 & -81 & 15 & -5.3827 \\
\hline & R Lingual gyrus & 183 & & & & \\
\hline & R Calcarine & 157 & & & & \\
\hline & L Lingual gyrus & 156 & & & & \\
\hline & L Cuneus & 76 & & & & \\
\hline & R Cuneus & 52 & & & & \\
\hline
\end{tabular}

Peak coordinates refer to the point with the highest $\mathrm{t}$ value in the cluster, not the specific region

Note: $x, y, z$ coordinates of peak locations in the Montreal Neurological Institute space (MNI)

ALFF Amplitude of low frequency fluctuations, HC Healthy control, SZ Schizophrenia patients, $L$ Left, $R$ Right

\section{Cognitive assessment outcome}

Compared to the $\mathrm{HC}$ group, the SZ group showed a significantly impaired total score and were impaired in all domains (Fig. 2).

\section{Correlations between ALFF and MCCB}

Both cluster F (Right paracentral lobule and postcentral gyrus) $(p<0.05, r=-0.42)$ and cluster I (Left postcentral gyrus and paracentral lobule) $(\mathrm{p}<0.05, r=-0.43)$ have a negative correlation with the MCCB symbol coding sub-test (M2) in SZ patients, results after the FDR correction $(\mathrm{q}<0.05)$ (Figs. 3 and 4$)$. When we use stricter significance levels of FDR correction or Bonferroni correction the results cannot hold. We also computed correlations in healthy samples, and there was a potentially positive correlation existed between the two brain regions and $\mathrm{MCCB}$ data without corrections (Additional file 1).

\section{Discussion}

The present study found cognitive impairments and ALFF differences between SZ patients and HC. Specifically, there was a negative correlation between the symbol-coding subtest and ALFF values in the bilateral postcentral gyri and the paracentral lobule in SZ patients.

We found that SZ patients showed cognitive impairments in all domains, which is consistent with previous studies that used the same cognitive measurement [15]. Deficits processing speed [33], working memory [34], verbal learning [7], reasoning/problem-solving [35], visual learning [36], and attention/vigilance [37] were found in SZ patients. Similar cognitive impairments were also reported in studies investigating patients with other psychiatric diagnoses [38-41]; however, the cognitive deficits were not as severe or as widespread as those seen in SZ patients. Therefore, the differences in the 


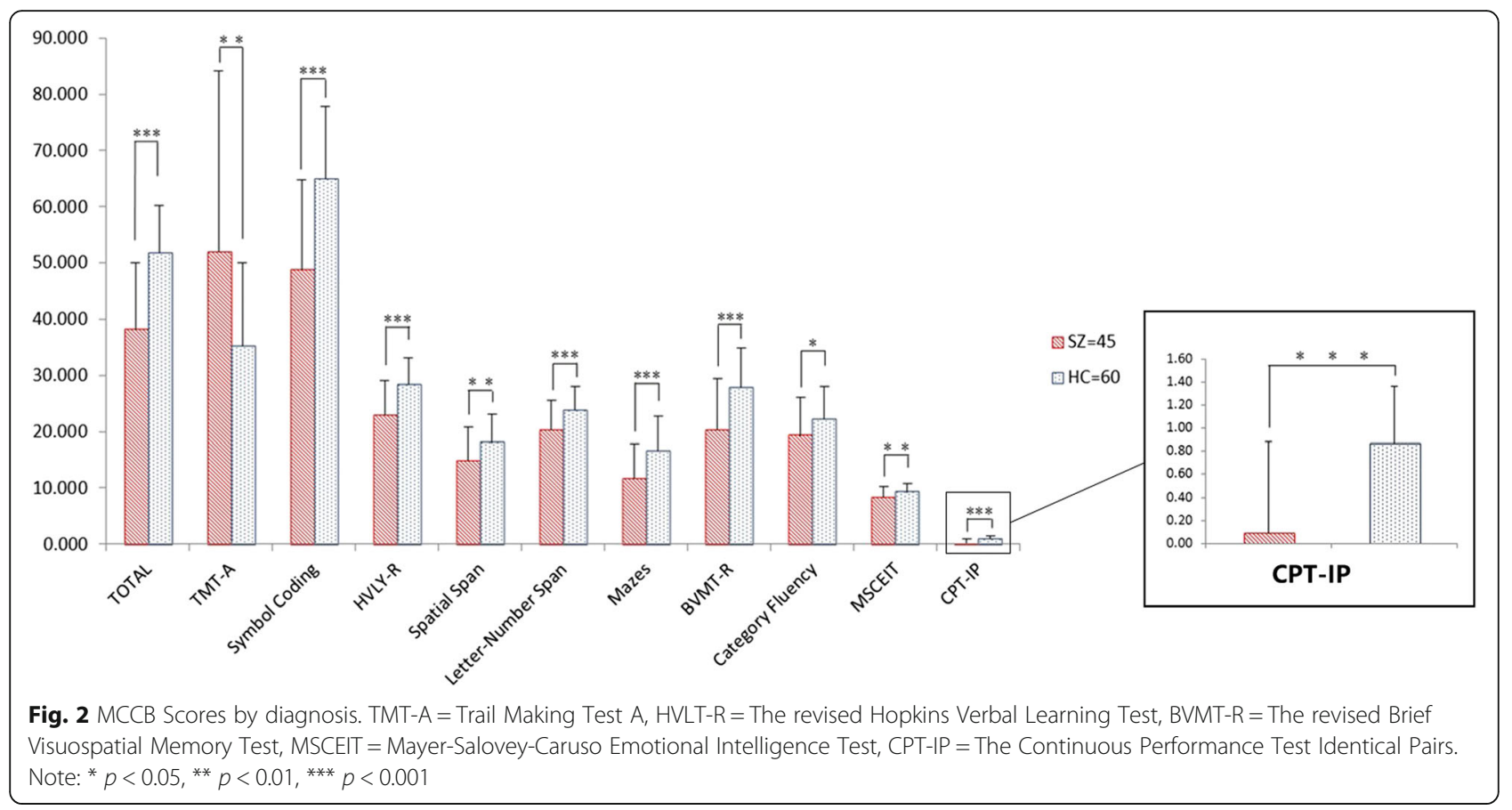

extent of cognitive impairment are likely due to the unique pathophysiological mechanisms of SZ. Our R-fMRI results showed increased ALFF in the bilateral caudate, the middle frontal gyri, and the left superior frontal gyrus, which is consistent with the established findings in SZ patients [42]. Tumbwene et al. [43] reported decreased ALFF changes similar to ours in the bilateral orbital inferior frontal gyri. A meta-analysis [27] also reported increased ALFF in the medial superior frontal gyri, which is consistent with our study. Furthermore, previous studies have reported decreased ALFF in the bilateral lingual [28], calcarine, cuneus, and postcentral gyri [44] areas of the brains of SZ patients (Table 3).
Previous studies reported ALFF changes in the hippocampus/parahippocampus [26], temporal pole, parahippocampal gyrus [28] and temporal fusiform [29], but these anomalies were not found in the present study; these differences may be due to population heterogeneity, different cultural backgrounds and different experimental parameters.

The symbol-coding subtest in the MCCB was used to evaluate processing speed in our study, which was also related to decreased ALFF in the bilateral postcentral gyri and paracentral lobule of SZ patients. These two areas are adjacent; the postcentral gyrus is located in the primary somatosensory cortex, and the paracentral

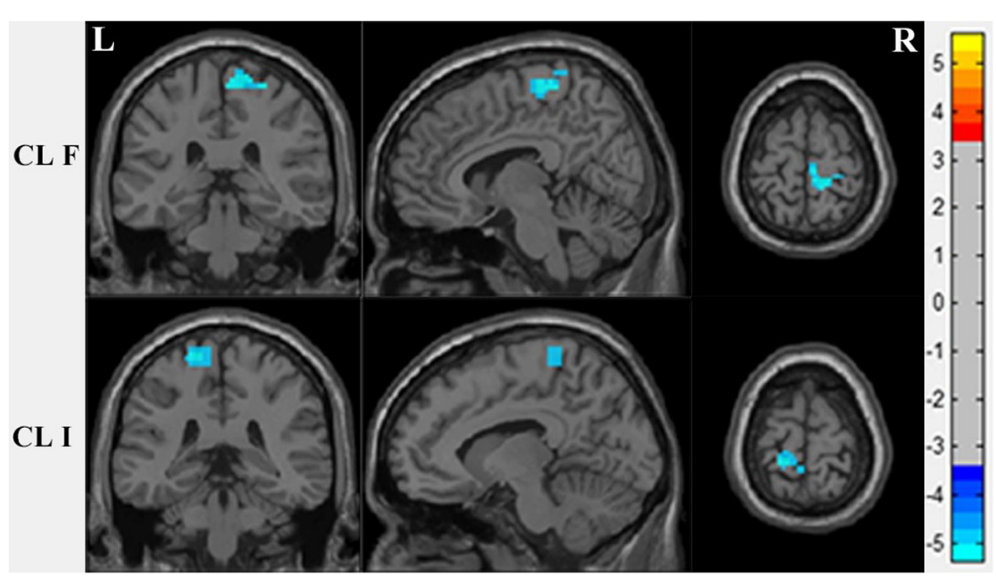

Fig. 3 These regions associated with symbol coding, after the FDR correction $(p<0.05)$. The color bar represents the range of $t$ values. $L$, left; $R$, right; $\mathrm{CL}$, cluster 

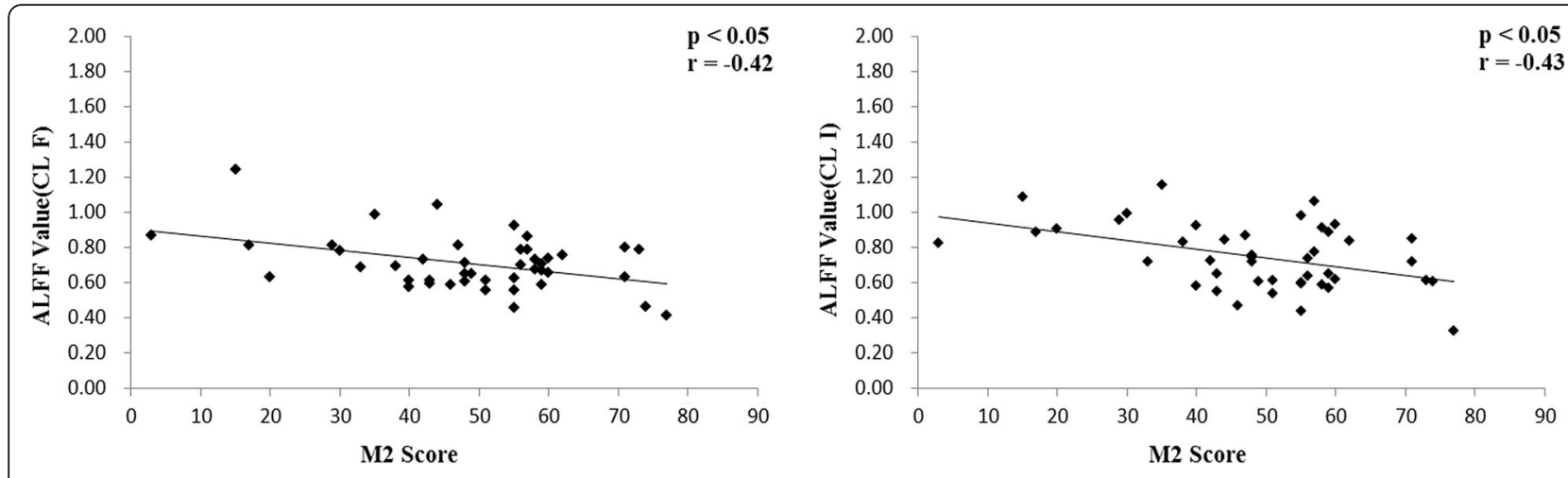

Fig. 4 Scatter diagram describing the relevant trends of cluster F and I, ALFF value, and the symbol coding score

lobule encompasses the medial continuation of the preand postcentral gyrus. The anterior region in the frontal lobe forms the supplementary motor area. Processing speed is important for SZ patients, and impairment in this area has been widely reported in previous studies $[13,15,33]$. Impairment in processing speed is an important factor for predicting the functional outcome of SZ patients [45-48]. Compared to other affected domains, processing speed exhibits the most serious deficit in SZ patients, with an average of approximately 1.5 standard deviations below that of $\mathrm{HC}$ [45]. The nature of the neuropsychological testing of processing speed requires participants to rapidly execute relatively simple tasks that integrate many basic cognitive operations. Therefore, it is not surprising that brain imaging studies on processing speed focused on reaction time. A previous task-state study reported [49] higher activity in the postcentral gyrus when subjects responded slower relative to previous responses. In the present study, activity in the postcentral gyrus in SZ patients was also negatively correlated with the speed of processing. Therefore, in this study, higher postcentral gyrus activity may lead to slower reaction times, which may impact processing speed in SZ patients. The somatosensory cortex is related to reaction times [50], and abnormalities in the postcentral gyrus, which is part of somatosensory cortex, may be the cause of longer reaction times in patients and may affect the speed of processing. Another region associated with processing speed is the bilateral paracentral lobule, which is part of the sensorimotor region. The paracentral lobules project to downstream motor nuclei and react to movements [51-54], which potentially contributes to motor function deficits in SZ patients and is associated with dysfunction and

Table 3 Overlaps between our study and previous resting-state functional Magnetic Resonance Imaging studies

\begin{tabular}{lll}
\hline & Our study & Previous study \\
\hline Increased ALFF & bilateral caudate & Turner et al. 2013 \\
& middle frontal gyri & Turner et al. 2013 \\
& left superior frontal gyrus & Turner et al. 2013 \\
& medial superior frontal gyri. & Xu et al. 2015 \\
Decreased ALFF & bilateral orbital inferior frontal gyri & Mwansisya et al. 2017 \\
& bilateral lingual & Alonso-Solis et al. 2017 \\
& bilateral calcarine & Salvador et al. 2017 \\
& bilateral cuneus & Salvador et al. 2017 \\
& bilateral postcentral gyri & Salvador et al. 2017 \\
Each article reported different & hippocampus & Previous literature reports were inconsistent in these ragion. \\
& parahippocampus & \\
& sensorimotor cortex & \\
& temporal pole & \\
& parahippocampal gyrus & \\
No clear report & temporal fusiform & \\
\hline
\end{tabular}


structural abnormalities in this region [55]. We also found potential positive correlations in healthy samples between these two brain regions and the symbol-coding subtest. However, these correlations disappeared with FDR correction $(\mathrm{q}<0.05)$. A previous study on fractional ALFF and cognition (attention) also found opposite trends in SZ vs HC [56]. We speculate that this correlation may be a special functional pattern of the brain in SZ patients after cognitive impairment.

We also observed increased ALFF in the bilateral pars triangularis inferior frontal gyrus of SZ patients, which has not been reported previously. The pars triangularis is part of Broca's region and plays a role in language and interpersonal information processing. The pars triangularis inferior frontal gyrus is related to the pathogenesis of positive symptoms, and gray matter volume reductions in this area may represent a vulnerability to SZ, which is considered to be a way to determine the developmental trajectory of disease [57]. However, the neurobiological basis for our finding remains unclear. Although there was no relationship between this area and the MCCB subtest in the present study, the significance of the anomalies in this region require further investigation.

There are some limitations in our research. Participants in the resting-state MRI study were required not to think during the scan, but this is an ideal state. Thinking may have affected the results of the experiment. This factor is a problem that remains unsolved. Increasing the sample size, retesting the same sample and measuring the stability of the index may be future solutions. We were unable to control the medications prescribed to our participants, thus some patients received medical treatment, while others did not; therefore, the effects of antipsychotics on cognitive function and ALFF cannot be discounted. Future studies should recruit people with schizophrenia who are initially untreated. Additionally, some data from the duration and BPRS (Brief Psychiatric Rating Scale) scores were missing despite our best efforts to collect all data; thus, our findings should be cautiously interpreted due to insufficient data from some participants. Our correlation results did not withstand stricter FDR significance levels or Bonferroni correction $(p<0.05)$. We cannot verify results on public SZ datasets because of the lack of fMRI and MCCB data.

\section{Conclusion}

Our results support the hypothesis of an underlying relationship between ALFF and cognition impairment (e.g., speed of processing) in SZ patients. In the present study, SZ patients displayed decreased ALFF in the bilateral postcentral gyri and paracentral lobule, and both areas may be associated with the symbol-coding subtest in SZ patients.

\section{Additional file}

Additional file 1: Supplementary materials. (DOCX 250 kb)

\begin{abstract}
Abbreviations
ALFF: Amplitude of low-frequency fluctuation; BPRS: Brief Psychiatric Rating Scale; EPI: Echo planar imaging; MNI: Montreal Neurological Institute;

FDR: False Discovery Rate; FFT: Fast Fourier Transform; GRF: Gaussian random field; HC: Healthy controls; MCCB: MATRICS consensus cognitive battery; R-

fMRI: Resting state functional magnetic resonance imaging; SCID-I: Structured Clinical Interview for DSM-IV Axis I Disorders; SD: Standard deviation;

SPSS: Statistical Product and Service Solutions; SZ: Schizophrenia
\end{abstract}

\section{Acknowledgements}

The authors wish to thank parents, advisor Tang \& Wang, all my classmates and colleagues who always encourage me but sometimes taunt me, the First Hospital of China Medical University, coffee and Earl Grey tea and Meten.

\section{Funding}

The authors were supported by research grants from the National Natural Science Foundation of China Funding support: National Natural Science Foundation of China (81571311, 81071099 and 81271499 to Y.T., 81725005, 81571331 to F.W.), National Science Fund for Distinguished Young Scholars (81725005 to F.W.), National Key Research and Development Program (2016YFC1306900 to Y.T.), Liaoning Education Foundation (Pandeng Scholar, F.W.) National Key Research and Development Program (2016YFC0904300 to F.W.), National High Tech Development Plan (863) (2015AA020513 to F.W.).

Availability of data and materials

The datasets used and/or analysed during the current study are available from the corresponding author on reasonable request.

\section{Authors' contributions}

$P W, J Y, Z Y, Y Z, M C, F W$ and $Y T$ designed the study. PW, YX, LL, XC, HL, JK, $X D, S W, Y Z$ and $X J$ were collected participants. PW, JD, RZ and JS did the analysis plan. PW drafted the manuscript. All authors read, contributed to and approved the final manuscript.

\section{Ethics approval and consent to participate}

The study was approved by the Medical Science Research Ethics Committee of the First Affiliated Hospital of China Medical University (approval reference number [2012]25-1). All participants have to give written informed consent before taking part in this study, the patients signed informed consent with the consent of their guardian, minor participants and their parents were all signed informed consent.

\section{Consent for publication}

Not applicable.

\section{Competing interests}

The authors declare that they have no competing interests.

\section{Publisher's Note}

Springer Nature remains neutral with regard to jurisdictional claims in published maps and institutional affiliations.

\section{Author details}

${ }^{1}$ Department of Psychiatry, The First Affiliated Hospital of China Medical University, Shenyang, Liaoning 110001, People's Republic of China. ${ }^{2}$ Brain Function Research Section, The First Affiliated Hospital of China Medical University, Shenyang, Liaoning 110001, People's Republic of China. ${ }^{3}$ Department of Gerontology, The First Affiliated Hospital of China Medical University, 155 Nanjing North Street, Heping District, Shenyang, Liaoning 110001, People's Republic of China. ${ }^{4}$ Department of Radiology, The First Affiliated Hospital of China Medical University, 155 Nanjing North Street, Heping District, Shenyang, Liaoning 110001, People's Republic of China. 
Received: 22 June 2018 Accepted: 18 December 2018 Published online: 17 January 2019

\section{References}

1. Lindenmayer JP, Fregenti S, Kang G, Ozog V, Ljuri I, Khan A, Goldring A, McGurk SR. The relationship of cognitive improvement after cognitive remediation with social functioning in patients with schizophrenia and severe cognitive deficits. Schizophr Res. 2017;185:154-60.

2. Lystad JU, Falkum E, Haaland VO, Bull H, Evensen S, McGurk SR, Ueland T. Cognitive remediation and occupational outcome in schizophrenia spectrum disorders: a 2year follow-up study. Schizophr Res. 2017;185:122-9.

3. Bora E. Neurodevelopmental origin of cognitive impairment in schizophrenia. Psychol Med. 2015;45:1):1-9.

4. Dorofeikova M, Neznanov N, Petrova N. Cognitive deficit in patients with paranoid schizophrenia: its clinical and laboratory correlates. Psychiatry Res. 2017;262:542-8.

5. Fett AK, Viechtbauer W, Dominguez MD, Penn DL, van Os J, Krabbendam L. The relationship between neurocognition and social cognition with functional outcomes in schizophrenia: a meta-analysis. Neurosci Biobehav Rev. 2011;35(3):573-88.

6. Croca M, Lagodka A, Gadel R, Bourdel MC, Bendjemaa N, Gaillard R, Olie JP, Champagne-Lavau M, Krebs MO, Amado I. Theory of mind and schizophrenia in young and middle-aged patients: influence of executive functions. Psychiatry Res. 2018;259:532-7.

7. Alexiadou A, Bozikas VP, Kosmidis MH, Parlapani E, Kiosseoglou G, Fokas K. The effect of impaired verbal memory retrieval on autobiographical memory across different life periods in schizophrenia. Compr Psychiatry. 2018;80:81-8.

8. Gomez-Benito J, Berrio Al, Guilera G, Rojo E, Purdon S, Pino O. The screen for cognitive impairment in psychiatry: proposal for a polytomous scoring system. Int J Methods Psychiatr Res. 2017;27:e1598.

9. Green MF, Nuechterlein KH, Gold JM, Barch DM, Cohen J, Essock S, Fenton WS, Frese F, Goldberg TE, Heaton RK, et al. Approaching a consensus cognitive battery for clinical trials in schizophrenia: the NIMH-MATRICS conference to select cognitive domains and test criteria. Biol Psychiatry. 2004;56(5):301-7.

10. Nuechterlein PD KH, Green PD MF, Kern PD RS, Baade PD LE, Barch PD DM, Cohen MD JD, Susan Essock PD, Fenton MD WS, Frese III PD FJ, Gold PD JM, et al. The MATRICS Consensus Cognitive Battery, Part 1:Test Selection, Reliability, and Validity. Am J Psychiatry. 2008;165(10):203-13.

11. Kern PD RS, Nuechterlein PD KH, Green PD MF, Baade PD LE, Fenton MD WS, Gold PD JM, Keefe PD RSE, Raquelle Mesholam-Gately PD, Jim Mintz PD, Seidman PD $\sqcup$, et al. The MATRICS Consensus Cognitive Battery, Part 2: Co-Norming and Standardization. Am J Psychiatry. 2008;165(6):214-20.

12. Kern RS, Green MF, Nuechterlein KH, Deng BH. NIMH-MATRICS survey on assessment of neurocognition in schizophrenia. Schizophr Res. 2004; 72(1):11-9.

13. Lewandowski KE, Whitton AE, Pizzagalli DA, Norris LA, Ongur D, Hall MH Reward learning, Neurocognition, social cognition, and symptomatology in psychosis. Frontiers in psychiatry. 2016;7:100.

14. Johnson MK, McMahon RP, Robinson BM, Harvey AN, Hahn B, Leonard CJ, Luck SJ, Gold JM. The relationship between working memory capacity and broad measures of cognitive ability in healthy adults and people with schizophrenia. Neuropsychology. 2013;27(2):220-9.

15. Kern RS, Gold JM, Dickinson D, Green MF, Nuechterlein KH, Baade LE, Keefe RS, Mesholam-Gately RI, Seidman LJ, Lee C, et al. The MCCB impairment profile for schizophrenia outpatients: results from the MATRICS psychometric and standardization study. Schizophr Res. 2011; 126(1-3):124-31.

16. Zou YZ, Cui JF, Wang J, Chen N, Tan SP, Zhang D, Xu Z, Song SG, Wang YH, $\mathrm{Li} Y$, et al. Clinical reliability and validity of the Chinese version of measurement and treatment research to improve cognition in schizophrenia consensus cognitive battery. Chin J Psychiatry. 2009;41(5): 29-33.

17. Zhang B, Han M, Tan S, De Yang F, Tan Y, Jiang S, Zhang X, Huang XF. Gender differences measured by the MATRICS consensus cognitive battery in chronic schizophrenia patients. Sci Rep. 2017;7(1):11821.

18. Shi C, He Y, Cheung EF, Yu X, Chan RC. An ecologically valid performancebased social functioning assessment battery for schizophrenia. Psychiatry Res. 2013;210(3):787-93.
19. Tan SP, Jie-Feng C, Fan FM, Zhao YL, Chen N, Fan HZ, Zhang JG, Wang YH, Yoon JH, Soares JC, et al. Smoking, MATRICS consensus cognitive battery and P50 sensory gating in a Han Chinese population. Drug Alcohol Depend. 2014;143:51-7.

20. Zang YF, He Y, Zhu CZ, Cao QJ, Sui MQ, Liang M, Tian LX, Jiang TZ, Wang YF. Altered baseline brain activity in children with $A D H D$ revealed by resting-state functional MRI. Brain Dev. 2007;29(2):83-91.

21. Cordes D, Haughton VM, Arfanakis K, Carew JD, Turski PA, Moritz CH, Quigley MA, Meyerand ME. Frequencies contributing to functional connectivity in the cerebral cortex in "resting-state" data. AJNR Am J Neuroradiol. 2001;22(7):1326-33.

22. Kiviniemi $\mathrm{V}$, Kantola $\mathrm{JH}$, Jauhiainen J, Tervonen O. Comparison of methods for detecting nondeterministic BOLD fluctuation in fMRI. Magn Reson Imaging. 2004;22(2):197-203.

23. Fox MD, Snyder AZ, Vincent JL, Raichle ME. Intrinsic fluctuations within cortical systems account for intertrial variability in human behavior. Neuron. 2007:56(1):171-84

24. Mennes M, Zuo XN, Kelly C, Di Martino A, Zang YF, Biswal B, Castellanos FX, Milham MP. Linking inter-individual differences in neural activation and behavior to intrinsic brain dynamics. Neuroimage. 2011;54(4):2950-9.

25. Zou Q, Ross TJ, Gu H, Geng X, Zuo XN, Hong LE, Gao JH, Stein EA, Zang YF, Yang $Y$. Intrinsic resting-state activity predicts working memory brain activation and behavioral performance. Hum Brain Mapp. 2013;34(12):3204-15.

26. Hoptman MJ, Zuo XN, Butler PD, Javitt DC, D'Angelo D, Mauro CJ, Milham MP. Amplitude of low-frequency oscillations in schizophrenia: a resting state fMRI study. Schizophr Res. 2010;117(1):13-20.

27. Xu Y, Zhuo C, Qin W, Zhu J, Yu C. Altered spontaneous brain activity in schizophrenia: a meta-analysis and a large-sample study. Biomed Res Int. 2015:2015:204628

28. Alonso-Solis A, Vives-Gilabert Y, Portella MJ, Rabella M, Grasa EM, Roldan A, Keymer-Gausset A, Molins C, Nunez-Marin F, Gomez-Anson B, et al. Altered amplitude of low frequency fluctuations in schizophrenia patients with persistent auditory verbal hallucinations. Schizophr Res. 2017;189:97-103.

29. Zheng J, Zhang Y, Guo X, Duan X, Zhang J, Zhao J, Chen H. Disrupted amplitude of low-frequency fluctuations in antipsychotic-naive adolescents with early-onset schizophrenia. Psychiatry Res. 2016;249:20-6.

30. Huang $X Q$, Lui $S$, Deng $W$, Chan $R C$, Wu QZ, Jiang $L$, Zhang JR, Jia ZY, $\mathrm{Li} X \mathrm{~L}$, Li F, et al. Localization of cerebral functional deficits in treatment-naive, first-episode schizophrenia using resting-state fMRI. Neurolmage. 2010;49(4):2901-6.

31. Yan CG, Wang XD, Zuo XN, Zang YF. DPABI: Data Processing \& Analysis for (resting-state) brain imaging. Neuroinformatics. 2016;14(3):339-51.

32. Andreasen NC, Pressler M, Nopoulos P, Miller D, Ho BC. Antipsychotic dose equivalents and dose-years: a standardized method for comparing exposure to different drugs. Biol Psychiatry. 2010;67(3):255-62.

33. Heinrichs RW, Pinnock F, Muharib E, Hartman L, Goldberg J, McDermid Vaz S. Neurocognitive normality in schizophrenia revisited. Schizophrenia research Cognition. 2015;2(4):227-32.

34. Trapp W, Dotterweich S, Hintner L, Wollny H, Lautenbacher S, Hajak G. Speed and capacity of working memory and executive function in schizophrenia compared to unipolar depression. Schizophr Res Cogn. 2017:10:1-6.

35. Mirian D, Heinrichs RW, McDermid Vaz S. Exploring logical reasoning abilities in schizophrenia patients. Schizophr Res. 2011:127(1-3):178-80.

36. Bodapati AS, Jenkins LM, Sharma RP, Rosen C. Visual memory uniquely predicts anhedonia in schizophrenia but not bipolar disorder. J Neuropsychol. 2017. https://doi.org/10.1111/jnp.12124.

37. Mohn C, Torgalsboen AK. Details of attention and learning change in firstepisode schizophrenia. Psychiatry Res. 2017;260:324-30.

38. Barch DM. Neuropsychological abnormalities in schizophrenia and major mood disorders: similarities and differences. Curr Psychiatry Rep. 2009;11(4): $313-9$.

39. Colin A, Depp PD, David J, Moore PD, David Sitzer PD, Barton W, Palmer PD, Lisa T, Eyler PD, Scott Roesch PD, Barry D, Lebowitz PD, Dilip V, Jeste MD. Neurocognitive Impairment in Middle-Aged and Older Adults with. Bipolar Disorder: Comparison to Schizophrenia and Normal. Comparison Subjects. J Affect Disord. 2007:103(1-3):201-9.

40. Mohn C, Rund BR. Neurocognitive profile in major depressive disorders: relationship to symptom level and subjective memory complaints. BMC psychiatry. 2016;16:108. 
41. Bo Q, Mao Z, Li X, Wang Z, Wang C, Ma X. Use of the MATRICS consensus cognitive battery (MCCB) to evaluate cognitive deficits in bipolar disorder: a systematic review and meta-analysis. PLoS One. 2017;12(4):e0176212.

42. Turner JA, Damaraju E, van Erp TG, Mathalon DH, Ford JM, Voyvodic J, Mueller BA, Belger A, Bustillo J, McEwen S, et al. A multi-site resting state fMRI study on the amplitude of low frequency fluctuations in schizophrenia. Front Neurosci. 2013;7:137.

43. Mwansisya TE, Hu A, Li Y, Chen X, Wu G, Huang X, Lv D, Li Z, Liu C, Xue Z, et al. Task and resting-state fMRI studies in first-episode schizophrenia: a systematic review. Schizophr Res. 2017;189:9-18.

44. Salvador R, Landin-Romero R, Anguera M, Canales-Rodriguez EJ, Radua J, Guerrero-Pedraza A, Sarro S, Maristany T, McKenna PJ, Pomarol-Clotet E. Non redundant functional brain connectivity in schizophrenia. Brain Imaging Behav. 2017;11(2):552-64.

45. Dickinson D, James M, Gold JM. a meta-analytic Comparison of digit symbol coding tasks and other cognitive measures in schizophrenia. Arch Gen Psychiatry. 2007;64(10):532-42.

46. Paolo Fusar-Poli M, Deste G, Smieskova R, Barlati S, Yung AR, Howes O, Stieglitz R-D, Vita A, McGuire P, Borgwardt S. Cognitive functioning in prodromal psychosis. Arch Gen Psychiatry. 2012;69(6):562-71.

47. Nuechterlein KH, Subotnik KL, Green MF, Ventura J, Asarnow RF, Gitlin MJ, Yee CM, Gretchen-Doorly D, Mintz J. Neurocognitive predictors of work outcome in recent-onset schizophrenia. Schizophr Bull. 2011;37(Suppl 2):S33-40.

48. Sanchez P. Predictors of longitudinal changes in schizophrenia: the role of processing speed. J Clin Psychiatry. 2009;70(6):888-96.

49. Bogler C, Vowinkel A, Zhutovsky P, Haynes JD. Default network activity is associated with better performance in a vigilance task. Front Hum Neurosci. 2017;11:623.

50. Alvaro P-L, Valls-Sole J, Wassermann EM, Brasil-Neto J, Hallett M. Effects of focal transcranial magnetic stimulation on simple reaction time to acoustic, VISUAL AND SOMATOSENSORY STIMULI. Brain : a journal of neurology. 1992;115:1045-59.

51. Havel P, Braun B, Rau S, Tonn JC, Fesl G, Bruckmann H, Ilmberger J. Reproducibility of activation in four motor paradigms. An fMRI study. J Neurol. 2006;253(4):471-6.

52. Picard N, Strick PL. Motor areas of the Medial Wall: a review of their location and functional activation. Cereb Cortex. 1996;6(11):342-53.

53. White LE, Andrews TJ, Hulette C, Richards A, Groelle M, Paydarfar J, Purves D. Structure of the human sensorimotor system. I: morphology and Cytoarchitecture of the central sulcus. Cereb Cortex. 1997;7(12):18-30.

54. Lim DSD SH, Pillay PK, Liiders H, Morris HH, Klem G, Wyllie IAA E. Functional anatomy of the human supplementary sensorimotor area: results of extraoperative electrical stimulation. Electroencephalogr Clin Neurophysiol. 1994;91(3):179-93.

55. Singh S, Goyal S, Modi S, Kumar P, Singh N, Bhatia T, Deshpande SN Khushu S. Motor function deficits in schizophrenia: an fMRI and VBM study. Neuroradiology. 2014;56(5):413-22.

56. He Z, Deng W, Li M, Chen Z, Jiang L, Wang Q, Huang C, Collier DA, Gong Q, Ma $X$, et al. Aberrant intrinsic brain activity and cognitive deficit in firstepisode treatment-naive patients with schizophrenia. Psychol Med. 2013; 43(4):769-80.

57. Iwashiro N, Suga M, Takano Y, Inoue H, Natsubori T, Satomura Y, Koike S, Yahata N, Murakami M, Katsura M, et al. Localized gray matter volume reductions in the pars triangularis of the inferior frontal gyrus in individuals at clinical high-risk for psychosis and first episode for schizophrenia. Schizophr Res. 2012;137(1-3):124-31.

\section{Ready to submit your research? Choose BMC and benefit from:}

- fast, convenient online submission

- thorough peer review by experienced researchers in your field

- rapid publication on acceptance

- support for research data, including large and complex data types

- gold Open Access which fosters wider collaboration and increased citations

- maximum visibility for your research: over $100 \mathrm{M}$ website views per year

At BMC, research is always in progress.

Learn more biomedcentral.com/submissions 